\section{Background and Context}

Unilateral tinnitus is a debilitating condition, with important psychosocial implications. ${ }^{1}$

- It must be investigated with a Magnetic Resonance Image (MRI) to rule out a rare but important finding of a cerebello-pontine angle lesion. ${ }^{2}$

- Good Practice Guidelines exist but are followed by $14 \%$ of GPs. ${ }^{3}$

- No widely accepted tinnitus management protocol $\rightarrow$ tinnitus patient pathway varies greatly. ${ }^{4}$

No previous systematic review evaluating existing literature relating to the tinnitus patient pathway.

\section{Objectives}

Primary aim: to identify practicing tinnitus patient pathways reported in the literature Secondary aim: establish the tools used a) to support changing the tinnitus pathway and $b$ ) to evaluate new tinnitus pathway effectiveness.

\section{Methods}

A systematic review was conducted of existing published literature to identify the most common unilateral tinnitus patient pathway within the UK.

- The Cochrane Database, Embase, Ovid MEDLINE, PubMed and NICE evidence search were searched in accordance with PRISMA guidelines.

- Search domains comprised "tinnitus" and 'patient pathway' along with related and exploded MESH terms.

- Included articles were assigned an evidence level in accordance with Centre for Evidence Based Medicine guidelines. Articles were critically appraised and methodological shortfalls identified in order to evaluate risk of bias within and across studies.

\section{Results:}

The systematic review found that the commonest NHS tinnitus patient pathway was as follows:

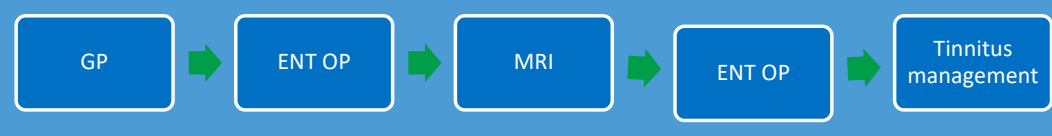

This is summarised in detail in Figure 3.

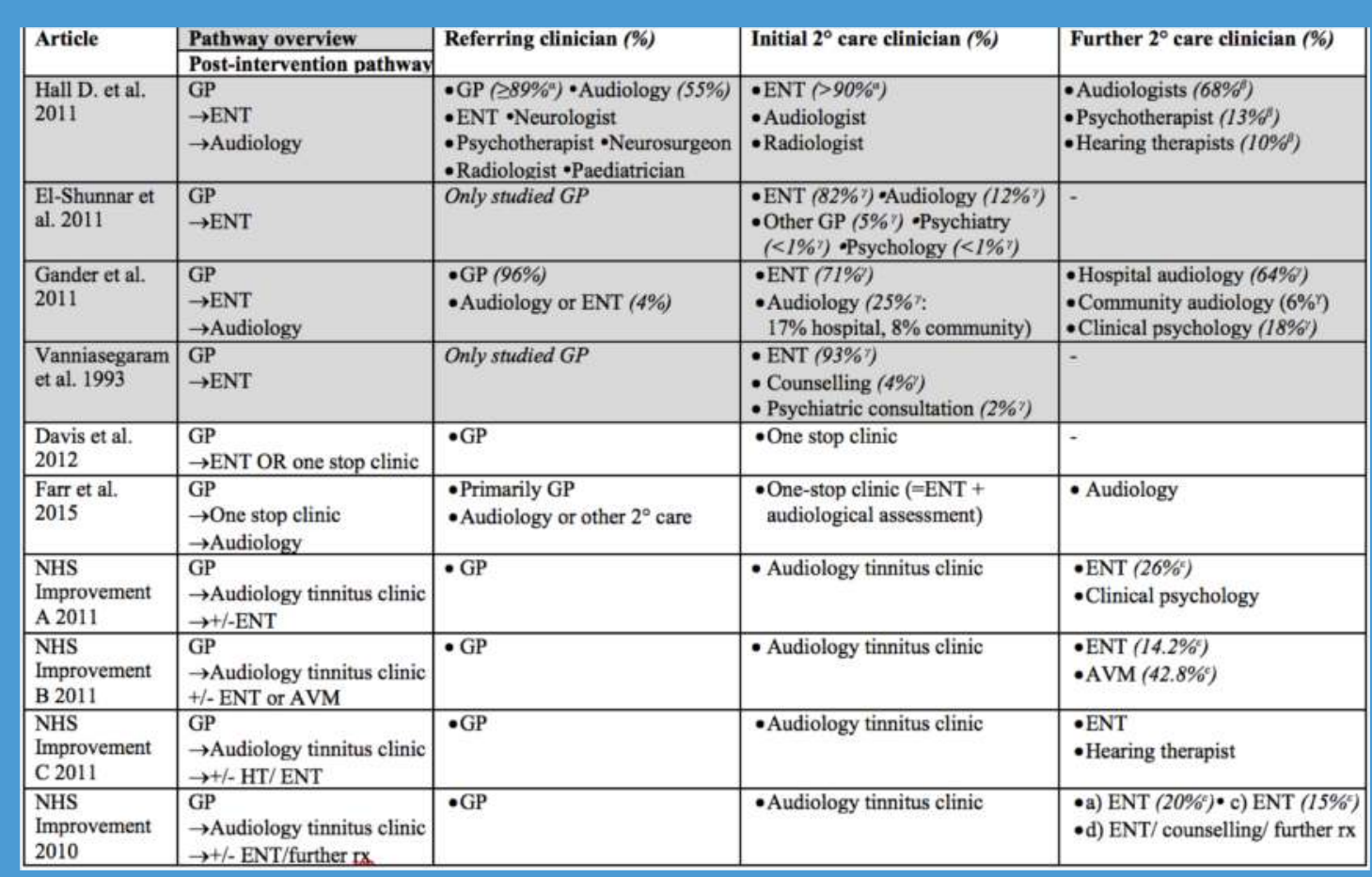

Figure 3: : The tinnitus pathway $-=$ not available, $2^{\circ}=$ secondary, $\alpha=$ across all countries (otherwise
UK), $\beta=\%$ of those referred on to further $2^{\circ} \mathrm{care}, \mathrm{V}=\%$ of referrals made to $2^{\circ}$ care from original UKe, $\beta=\%$ of those referred on to further $2^{\circ} \mathrm{care}, \mathrm{Y}=\%$ of referrals made to $2^{\circ}$ care from original
refering clinician, $\varepsilon=\%$ of those seen in secondary care, a,b,c,d see table $1, \mathrm{AVM}=$ Audiovestibular
Included studies

Of 8,176 records identified, 8 articles met the inclusion criteria (Figure 1). Articles included four descriptive cross-sectional surveys and six observational cohort studies, as summarized in (Figure 2).

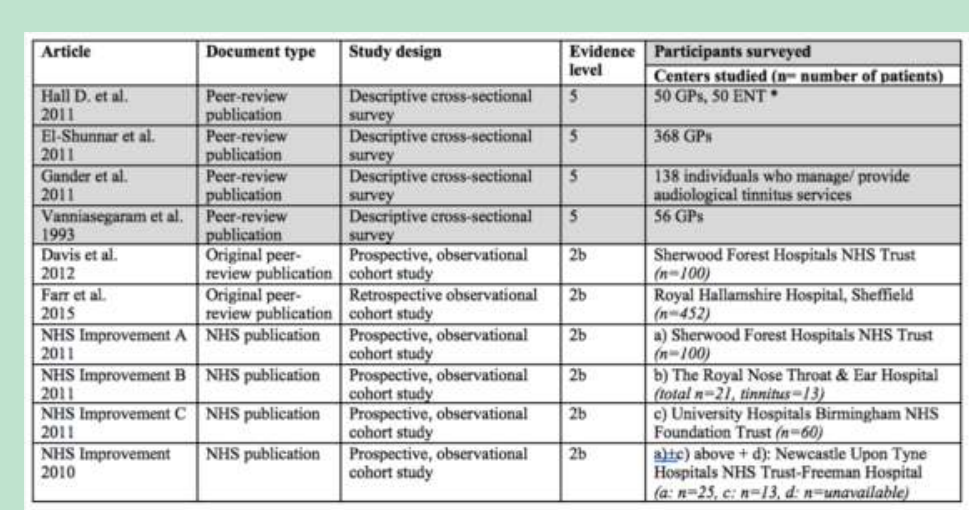

Figure 2: Summary of included articles ENT=Ear Nose and Throat, GP $=$ General Practitioner, NHS=National Health Service, *international study, UK data subset

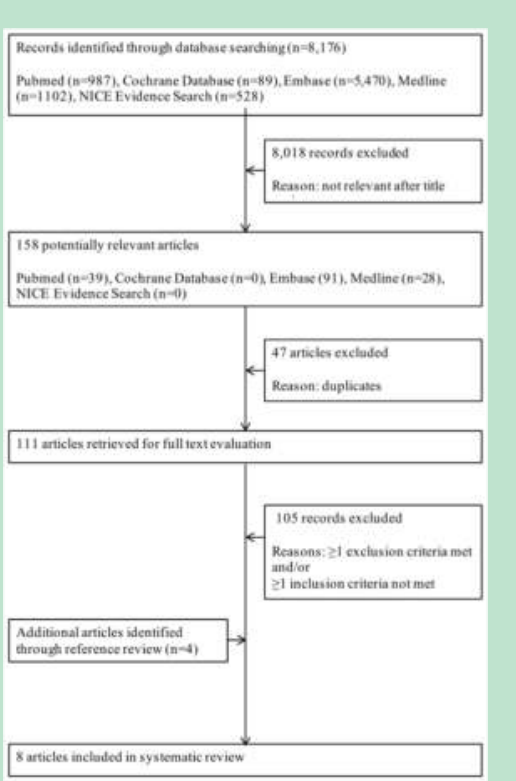

Figure 1: Flow chart of studies included in
Tools used to build a case for change included lean management techniques, patient involvement and retrospective audit (Figure 4).

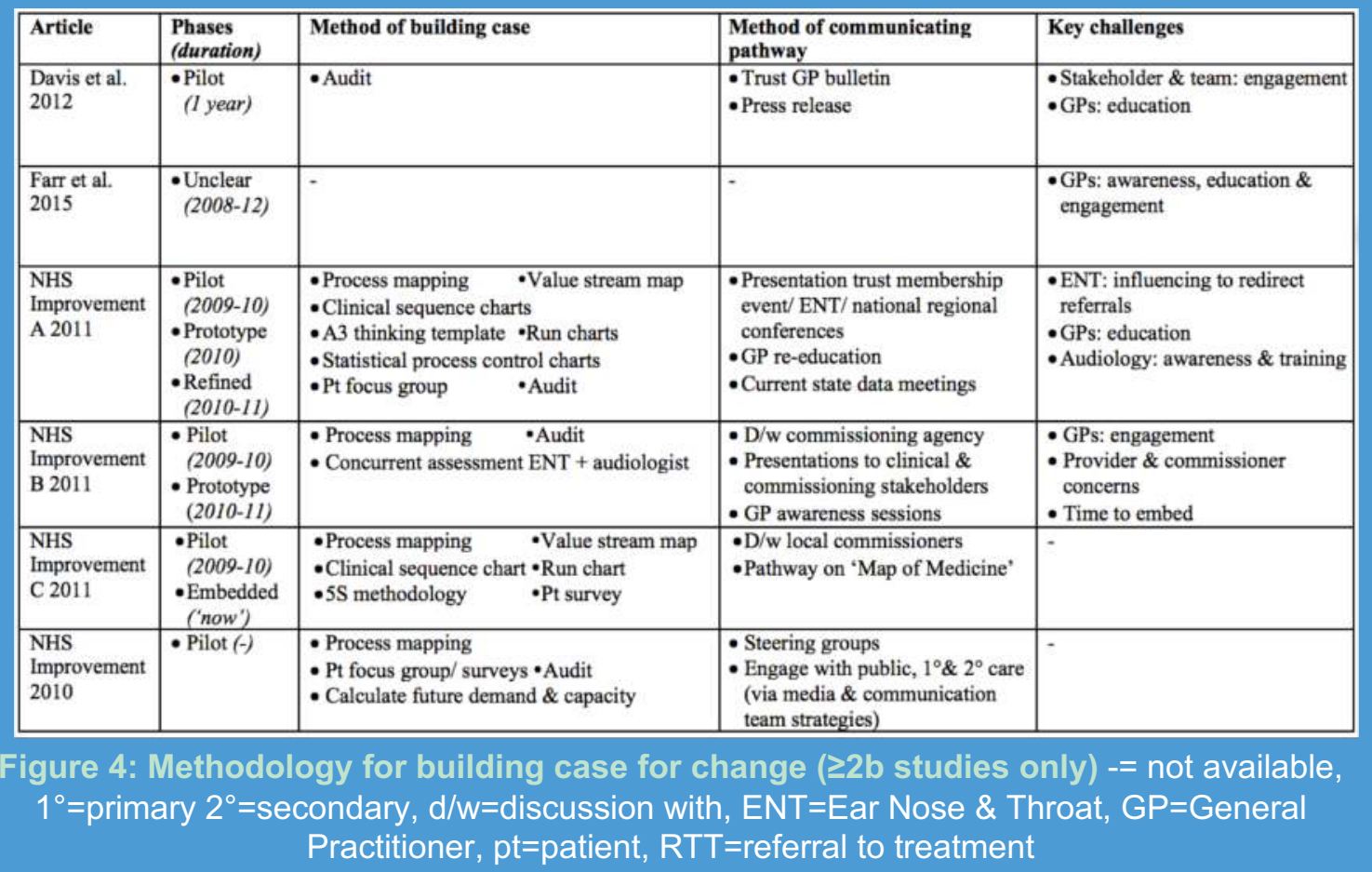

- New pathways were evaluated using clinical outcome measures (e.g. Tinnitus Handicap Inventory), process measures (e.g. referral to treatment), defect measures (e.g. inappropriate referrals) and patient satisfaction measures (Figure 5).

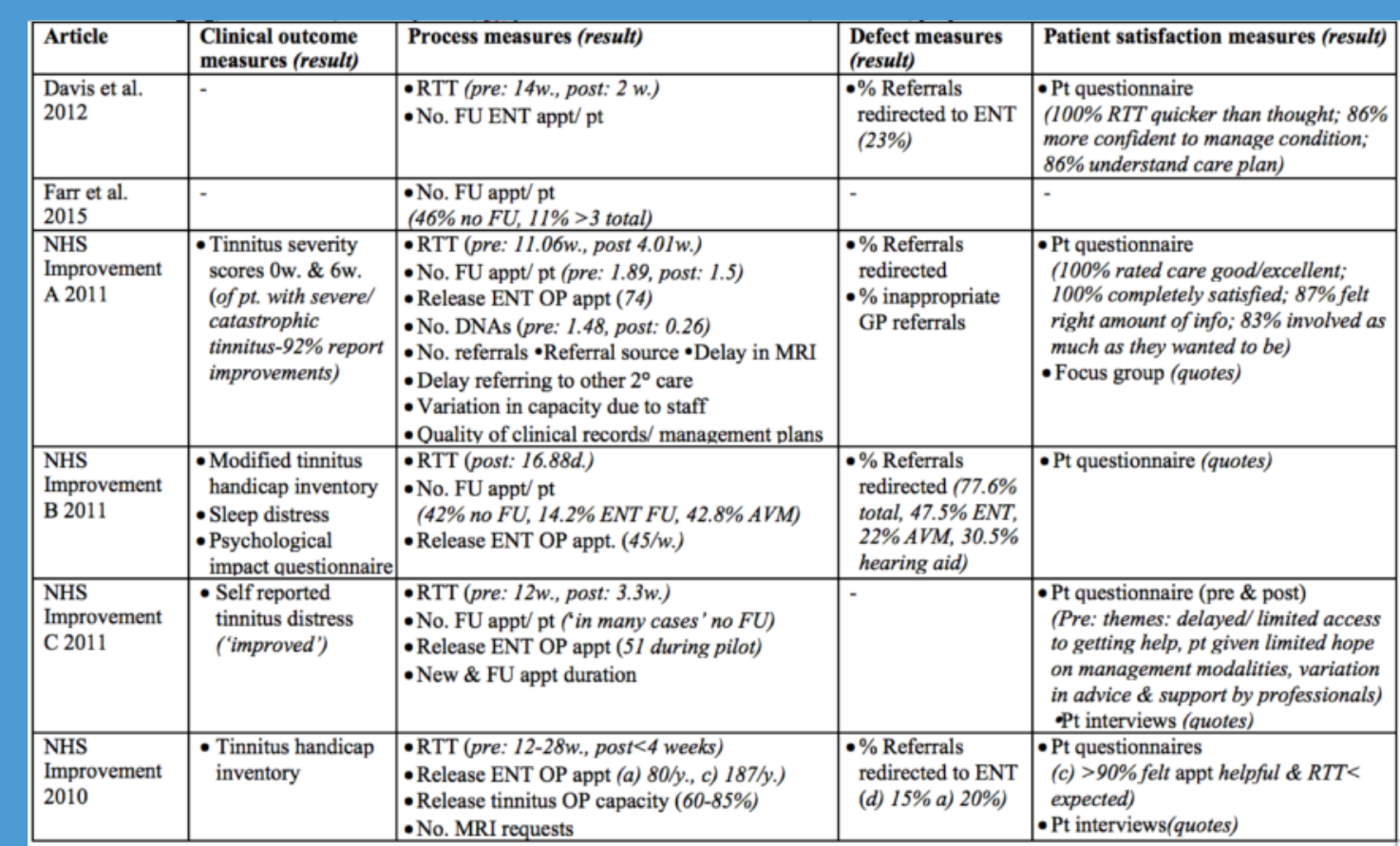

Figure 5: Evaluation of streamlined tinnitus pathway ( $\geq 2 \mathrm{~b}$ studies only) $-=$ not available,

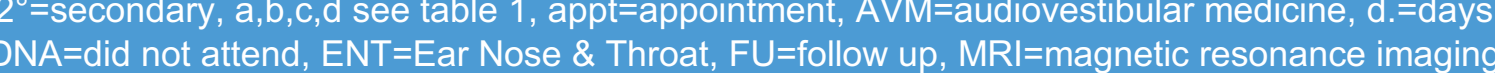

Medicine, ENT=Ear Nose and Throat, GP=General Practice, HT=Hearing Therapist, rx=treatment

\title{
Conclusions
}

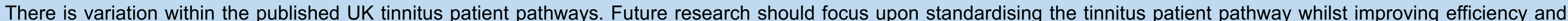

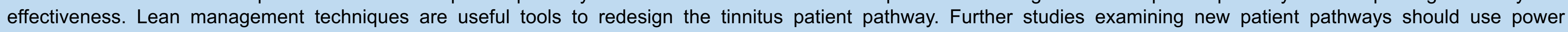
calculations, well-defined pre-intervention patient groups and a broad range of efficacy parameters.

References: 1) DOI: 10.3109/14992020309056079 2) PMID: 1935877 3) PMID: 21707872 4) PMID 22053947

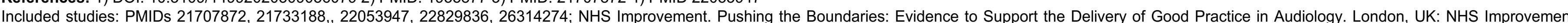

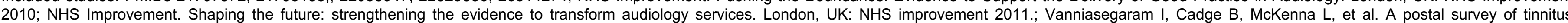
management in general practice. Journal of Audiological Medicine 1993;2(1). 